\title{
ESTRUTURA TRÓFICA E RELAÇÕES ECOLÓGICAS NA ICTIOFAUNA ASSOCIADA AO NAUFRÁGIO GONÇALO COELHO, SERRAMBI - PE
}

\author{
MARINS, Y.O. ${ }^{1 *}$; OLIVEIRA, P.G.V. ${ }^{1} \&$ RIBAS, C.A. ${ }^{2}$ \\ 1. Universidade Federal Rural de Pernambuco, Programa de Pós-Graduação em Recursos Pesquei- \\ ros, Recife, PE, Brasil. \\ 2. Universidade Federal de Pernambuco, Departamento de Oceanografia, Recife, PE, Brasil. \\ *Corresponding author: uripescapa@gmail.com
}

\begin{abstract}
Marins, Y.O.;Oliveira, P.G.V. \& Ribas, C.A. (2015). Estrutura trófica e relações ecológicas na ictiofauna associada ao naufrágio Gonçalo Coelho, Serrambi - PE. Braz. J. Aquat. Sci. Technol. 19(2). elSSN 1983-9057. DOI: 10.14210/ bjast.v19n2. Artificial structures are installed in aquatic environments with many objectives, like reversing the impacts of the human activities on natural reef environments and increasing productivity of marine organisms. It is expected that these constructs have the capacity to become similar or even perform the same functions of the natural reef environments. This ability directly affects the behavior, the ecological relationships and distribution of associated species. The present study aimed to observe and describe the feeding habits and the ecological relationships performed between species identified. The occurrence of planktivorous species was the predominant. This factor may be linked to the fact that the sinking function as a barrier to the currents provides the resuspension of nutrients in contact with the substrate. Significant ecological relationships linked to the power were also observed, mainly cleaning, that is a relationship of fundamental importance to keeping the health of the species that were found. Other important relations as follower behavior, formation of mixed shoal, predation and oophagy were also observed.
\end{abstract}

Key Words: Oophagy, Artificial reef, Reef fishes, Predation.

\section{INTRODUÇÃO}

Ambientes recifais são locais onde são encontradas as maiores diversidades de espécies entre os ecossistemas marinhos, abrigando algo em torno de $25 \%$ das espécies de peixes já descritas (Spalding et al., 2001). A grande variedade de habitats nesses ecossistemas é um dos fatores que mais contribuem para a alta diversidade (Sale, 1980), sendo a organização das comunidades ictíicas nesses ambientes diretamente relacionadas à estrutura física do habitat.

Com o aumento do impacto causado pelo homem a esses ecossistemas, vários países ao redor do mundo passaram a criar programas de implementação de recifes artificiais com objetivo de recuperar áreas degradadas (Seaman, 2000). Esses recifes artificiais podem ser definidos como um ou mais objetos, de origem natural ou humana, depositados propositalmente no ambiente marinho, visando influenciar processos físicos, biológicos ou socioeconômicos, relacionados aos próprios recursos (Seaman \& Jensen, 2000).

Em recifes artificiais, espera-se que relações ecológicas entre as espécies ocorram de forma similar aos recifes naturais, visto que essas estruturas visam desempenhar funções ecológicas semelhantes, além de servirem de abrigo contra predadores, áreas de reprodução e crescimento (Collins et al., 1991, Sale et al., 2005).
Em ambientes naturais, os peixes podem ocupar todos os níveis em uma cadeia trófica, que vão desde espécies herbívoras até os carnívoros (Keenleyside, 1979). Em recifes artificiais, espera-se que a ictiofauna recifal seja capaz de estabelecer comportamentos semelhantes aos encontrados em ambientes naturais, como territorialismo, agregação reprodutiva, formações de cardumes mistos, comportamentos seguidores e alimentação.

Dentre os comportamentos associados às atividades tróficas, a simbiose de limpeza entre os peixes limpadores e seus clientes é de fundamental importância, pois os mesmos removem ectoparasitas, tecido doente, escamas e muco provenientes de outras espécies, assegurando assim a saúde dos peixes (DeLoach, 1999, Arnal et al., 2001). Tais comportamentos são de grande importância devido a sua relevância na regulação das comunidades ictíicas recifais, em sua diversidade e abundância.

O presente estudo analisou a estrutura trófica da comunidade ictíica associada ao naufrágio Gonçalo Coelho, além das atividades simbióticas entre as espécies, como alimentação, comportamento seguidor, predação e atividades de limpeza, além de registrar também atividades reprodutivas. Essa análise comportamental é de extrema relevância em razão da sua grande importância no equilíbrio da diversidade e abundância dessas comunidades ictíicas. 


\section{MATERIAL E MÉTODOS}

\section{Caracterização da área e coleta de dados}

O Estado de Pernambuco situa-se no nordeste brasileiro, nas coordenadas geográficas de $07^{\circ} 15^{\prime}$

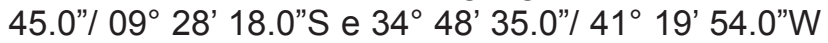
(Figura 01). Possui uma faixa litorânea de 180 km, com áreas de manguezais, bancos de fanerógamas, recifes costeiros e praias arenosas. Possui um clima tropical úmido. A profundidade na plataforma continental é de aproximadamente 50 metros de profundidade.
A temperatura da água varia entre $26^{\circ} \mathrm{C}$ e $30^{\circ} \mathrm{C}$. Os ventos predominantes são de leste-sudeste, com velocidade entre 3 e $5 \mathrm{~m} / \mathrm{s}$. A salinidade varia de 32,0 a 36,5 ppt (Cavalcanti \& Kempf, 1970).

O naufrágio Gonçalo Coelho (Tabela 01), está situado ao sul do Estado, na altura da Praia de Serrambi (Figura 01), município de Ipojuca. Distante da costa cerca de 08 milhas náuticas (aproximadamente $16 \mathrm{~km})$. Seu afundamento aconteceu no dia 29 de dezembro de 1999.

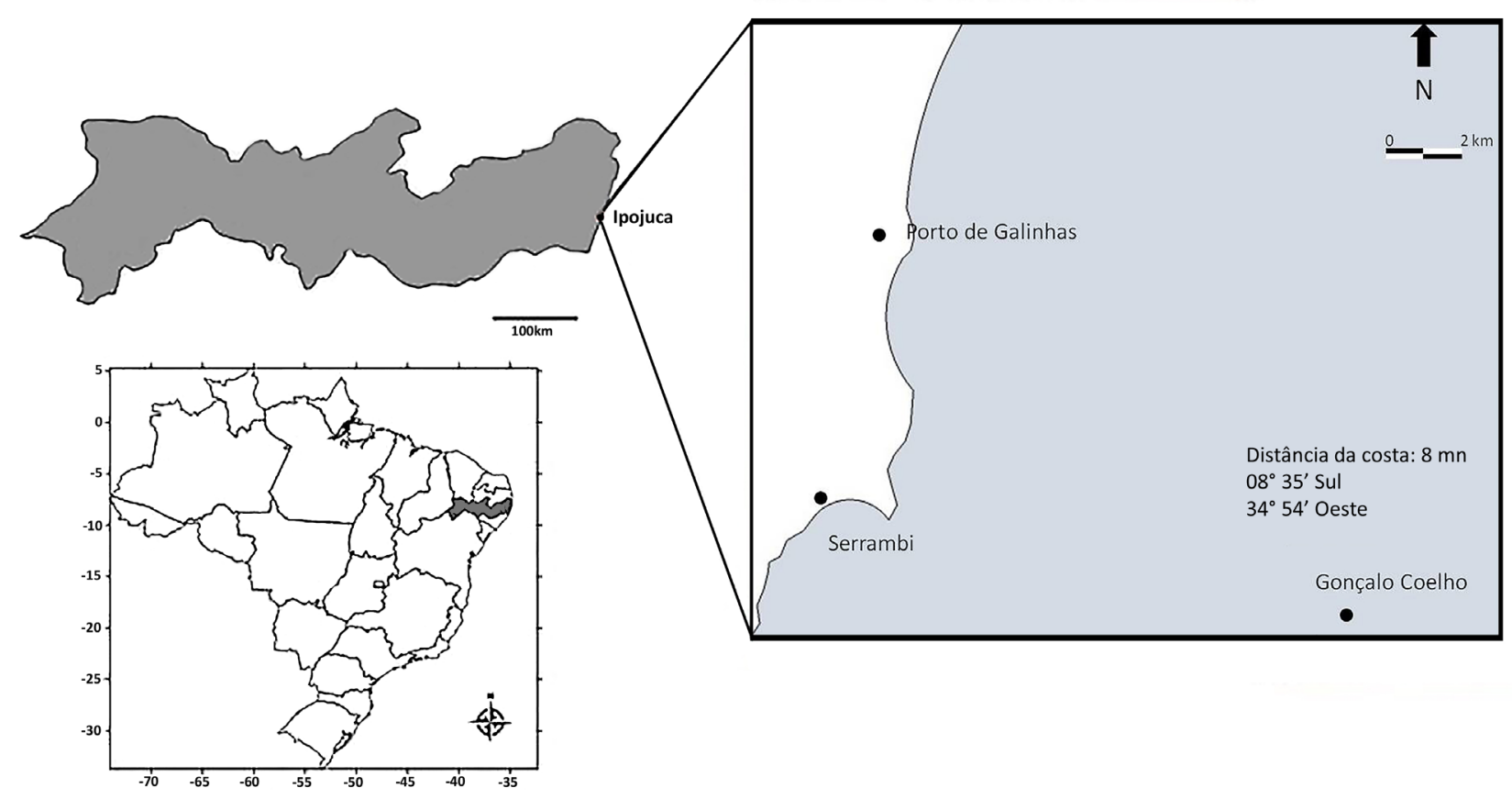

Figura 01 - Localização do naufrágio Gonçalo Coelho, Serrambi/PE.

Tabela 01 - Dados da embarcação Gonçalo Coelho utilizada como recife artificial para criação de novos pontos de mergulho na costa do Estado de Pernambuco. Fonte: Jr. Palazzo, 2011.

\section{Dados da embarcação}

Comprimento:

Data do afundamento:

Local:

Latitude:

Longitude:

Distância da costa:

Profundidade:

Motivo do afundamento:
$64 \mathrm{~m}$ - Boca: $8 \mathrm{~m}$

29/12/1999

Serrambi UF: PE

$08^{\circ} 35^{\prime}$ SUL

$034^{\circ} 54^{\prime}$ OESTE

08 milhas náuticas

$34 \mathrm{~m}$

Ponto de mergulho 


\section{Coleta de dados}

Entre os meses de novembro de 2011 e março de 2013, foram realizados 13 mergulhos autônomos, entre 10:00 da manhã e 13:00 da tarde, com auxílio de 03 mergulhadores, totalizando em 957 minutos de observações subaquáticas. Não ocorreram mergulhos durante os meses de maio, junho, julho e agosto de 2012 devido as fortes chuvas e ventos, o que impossibilitou a operação de mergulho na região nesse período. Foram realizados censos estacionários, para registro das interações ecológicas, além de buscas intensivas. Os mergulhadores distribuíram-se um por bombordo e outro por boreste, enquanto um terceiro percorria a porção superior (convés e casario) registrando as interações entre as espécies, ambos por meio de fotografias e vídeos, além de anotações em pranchetas de PVC.

\section{Estruturas tróficas}

Para a determinação dos hábitos alimentares, foi utilizada a classificação sugerida por Ferreira et al. (2004), onde as espécies foram divididas em 8 categorias tróficas, sendo: carnívoras generalistas $(C)$, piscívoros $(P)$, herbívoros territorialistas $(\mathrm{TH})$, herbívoros não-territorialistas $(\mathrm{RH})$, onívoros $(\mathrm{O})$, planctívoros (PL), predadores de invertebrados sésseis (SI), predadores de invertebrados móveis (MI).

Para as classes tróficas, foram realizados testes não paramétricos de Kruskal-Wallis, devido a distribuição não normal dos dados de abundância numérica das espécies. Os testes foram realizados utilizando-se o pacote estatístico XLSTAT 2013 para Windows.

\section{RESULTADOS}

\section{Estrutura trófica}

A ictiofauna associada ao naufrágio Gonçalo Coelho (Tabela 02), apresentou uma distribuição das categorias tróficas em que a mais abundante categoria foi a de peixes planctívoros (PL) $(54,13 \%)$ representada em sua maioria pelas espécies Chromis multilineta, Clepticus brasiliensis e Decapterus macarellus. Em seguida vieram os predadores de invertebrados móveis (MI) com 27,78\%, composta em sua maioria pelas espécies da família Haemulidae, Haemulon aurolineatum e Haemulon squamipinna, além de Bodianus rufus. Os onívoros (O) vieram logo em seguida com $15,09 \%$, composta em sua maioria por Abudefduf saxatilis. Em seguida vieram as categorias menos abundantes, com carnívoros (C) $1,64 \%$ da abundância, seguido dos predadores de invertebrados sésseis ( $\mathrm{SI}$ ) com $0,74 \%$, herbívoros não-territorialistas $(\mathrm{RH})$ com $0,35 \%$, piscívoros $(\mathrm{P})$ com $0,27 \%$ e herbívoros territorialistas $(\mathrm{TH})$ com apenas $0,001 \%$ sendo representada apenas pela espécie Stegastes pictus. Houve diferença significativa em relação à abundância total dos indivíduos entre as classes tróficas $(K=89,11$; $p<0,0001 . \alpha=0,05)$ (Figura 02).

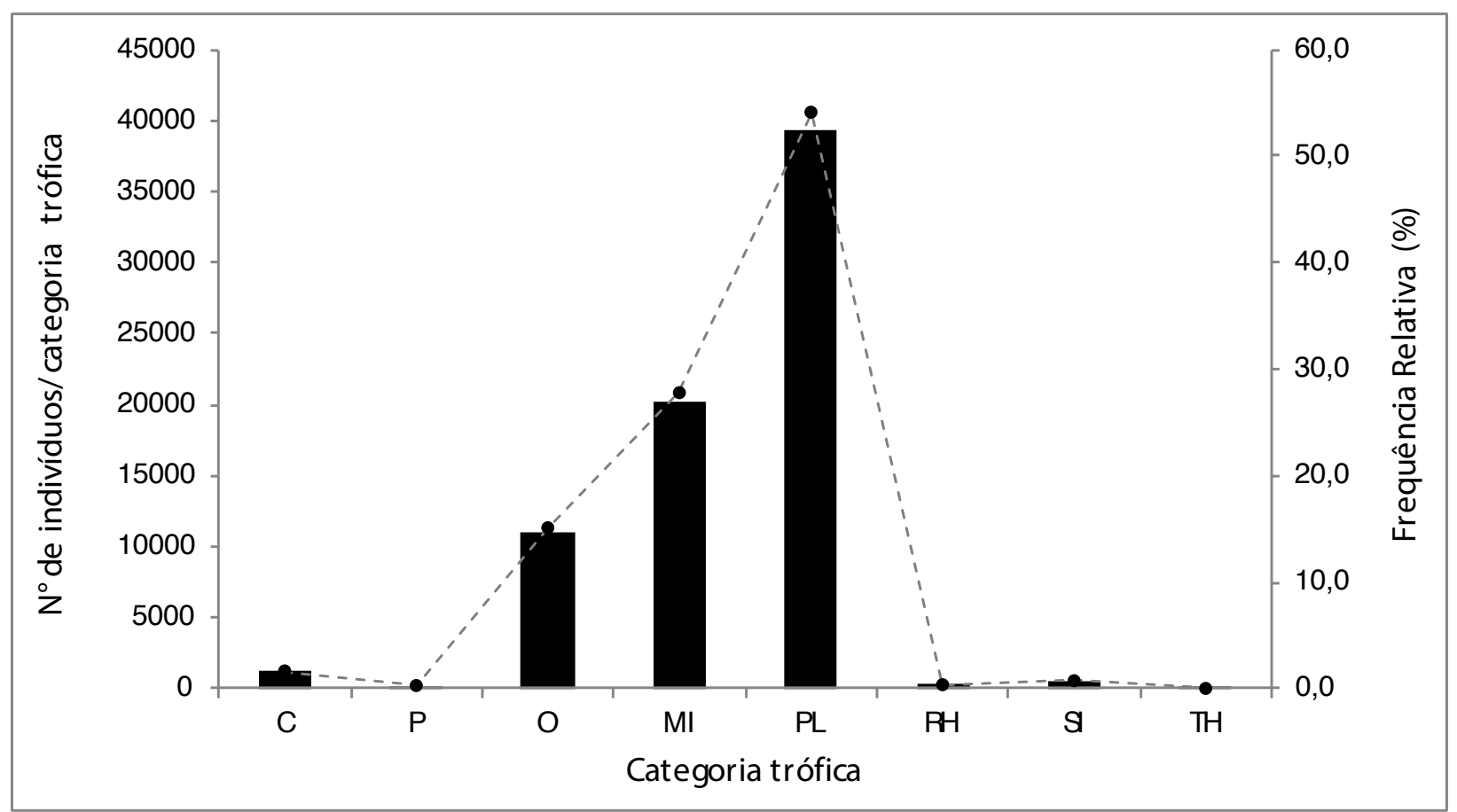

Figura 02 - Número de indivíduos (linha tracejada) e Frequência relativa (\%) (barras) por categoria trófica das espécies associadas ao naufrágio Gonçalo Coelho, Serrambi/PE. Categorias tróficas: carnívoros (C); piscívoros (P); onívoros (O); predadores de invertebrados móveis (MI); planctívoros $(\mathrm{PL})$; herbívoros não-territorialistas $(\mathrm{RH})$; predadores de invertebrados sésseis (SI); herbívoros territorialistas $(\mathrm{TH})$, segundo Ferreira et al. 2004. 
Tabela 02 - Lista de espécies com respectivas Frequência Relaticas (Fr\%) e Categorias Tróficas (C.T), registradas no naufrágio Gonçalo Coelho, Serrambi/PE. Categorias trófica: carnívoros (C); piscívoros (P); onívoros (O); predadores de invertebrados móveis (MI); planctívoros (PL); herbívoros não-erritorialistas $(\mathrm{RH})$; predadores de invertebrados sésseis (SI); herbívoros territorialistas (TH), segundo Ferreira et al. (2004).

\begin{tabular}{|c|c|c|c|}
\hline Família & Espécie & $\operatorname{Fr}(\%)$ & C.T \\
\hline \multicolumn{4}{|c|}{ Dasyatidae } \\
\hline & Dasyatis americana Hilderande \& Schoreder, 1928 & 0,029 & $\mathrm{C}$ \\
\hline \multicolumn{4}{|c|}{ Synodontidae } \\
\hline & Synodus intermedius (Spix \& Agassiz, 1829) & 0,029 & $\mathrm{P}$ \\
\hline \multicolumn{4}{|c|}{ Ogcocephalidae } \\
\hline & Ogcocephalus vespertilio (Linnaeus, 1758) & 0,004 & MI \\
\hline \multicolumn{4}{|c|}{ Holocentridae } \\
\hline & Holocentrus adscensionis (Osbeck, 1765) & 0,441 & MI \\
\hline & Myripristis jacobus Cuvier, 1829 & 3,536 & PL \\
\hline \multicolumn{4}{|c|}{ Grammistidae } \\
\hline & Rypticus saponaceus (Bloch \& Schneider, 1801) & 0,004 & $\mathrm{C}$ \\
\hline \multicolumn{4}{|c|}{ Epinephelidae } \\
\hline & Cephalopholis fulva (Linnaeus, 1758) & 0,495 & $\mathrm{C}$ \\
\hline & Epinephelus adscensionis (Osbeck, 1765) & 0,066 & $\mathrm{C}$ \\
\hline & Epinephelus itajara Lichtenstein, 1822 & 0,033 & $\mathrm{C}$ \\
\hline & Cephalopholis furcifer (Valenciennes, 1828) & 0,049 & PL \\
\hline \multicolumn{4}{|c|}{ Grammatidae } \\
\hline & Gramma brasilensis Sazima, Moura \& Rosa, 1997 & 0,008 & MI \\
\hline \multicolumn{4}{|c|}{ Opistognathidae } \\
\hline & Opistognathus cf.aurifrons (Jordan \& Thompson, 1905) & 0,004 & PL \\
\hline \multicolumn{4}{|c|}{ Apogonidae } \\
\hline & Apogon americanus Castelnau, 1855 & 0,008 & PL \\
\hline \multicolumn{4}{|c|}{ Malacanthidae } \\
\hline & Malacanthus plumieri Bloch, 1786 & 0,037 & $\mathrm{C}$ \\
\hline \multicolumn{4}{|c|}{ Echeneidae } \\
\hline & Echeneis naucrates Linnaeus, 1758 & 0,008 & $\mathrm{C}$ \\
\hline \multicolumn{4}{|c|}{ Carangidae } \\
\hline & Carangoides bartholomaei (Cuvier, 1833) & 1,847 & $\mathrm{O}$ \\
\hline & Caranx crysos (Mitchill, 1815) & 0,074 & $\mathrm{P}$ \\
\hline & Caranx latus Agassiz, 1831 & 0,499 & $\mathrm{P}$ \\
\hline & Decapterus macarellus (Cuvier, 1833) & 44,249 & PL \\
\hline & Decapterus punctatus (Cuvier, 1829) & 0,008 & PL \\
\hline & Elagatis bipinnulata (Quoy \& Gaimard, 1825) & 0,016 & $\mathrm{P}$ \\
\hline & Selene vomer (Linnaeus, 1758) & 2,553 & $\mathrm{C}$ \\
\hline
\end{tabular}




\begin{tabular}{|c|c|c|c|}
\hline Família & Espécie & $\operatorname{Fr}(\%)$ & C.T \\
\hline \multicolumn{4}{|c|}{ Lutjanidae } \\
\hline & Lutjanus alexandrei Moura \& Linderman, 2007 & 0,705 & $\mathrm{C}$ \\
\hline & Lutjanus cyanopterus (Cuvier, 1828) & 0,008 & $\mathrm{P}$ \\
\hline & Lutjanus analis (Cuvier, 1828) & 0,037 & $\mathrm{C}$ \\
\hline & Lutjanus jocu (Bloch \& Schneider, 1801) & 0,157 & $\mathrm{C}$ \\
\hline & Lutjanus synagris (Linnaeus, 1758) & 0,359 & $\mathrm{C}$ \\
\hline & Ocyurus chrysurus (Bloch, 1791) & 0,388 & $\mathrm{C}$ \\
\hline \multicolumn{4}{|c|}{ Haemulidae } \\
\hline & Anisotremus virginicus (Linnaeus, 1758) & 0,532 & MI \\
\hline & Anisotremus surinamensis (Bloch, 1791) & 0,450 & MI \\
\hline & Haemulon aurolineatum (Cuvier, 1830) & 38,125 & MI \\
\hline & Haemulon parra (Desmarest, 1823) & 3,415 & MI \\
\hline & Haemulon plumieri (Lacepède, 1801) & 1,089 & MI \\
\hline & Haemulon squamipinna Rosa \& Rosa, 1999 & 4,371 & MI \\
\hline \multicolumn{4}{|c|}{ Sciaenidae } \\
\hline & Odontoscion dentex (Cuvier, 1830) & 0,062 & $\mathrm{C}$ \\
\hline \multicolumn{4}{|c|}{ Mullidae } \\
\hline & Mulloidichthys martinicus (Cuvier, 1829) & 4,738 & MI \\
\hline & Pseudupeneus maculatus (Bloch, 1793) & 0,103 & MI \\
\hline \multicolumn{4}{|c|}{ Pempheridae } \\
\hline & Pempheris schomburgkii Müller \& Troschei, 1848 & 14,681 & PL \\
\hline \multicolumn{4}{|c|}{ Kyphosidae } \\
\hline & Kyphosus sp. & 0,058 & RH \\
\hline \multicolumn{4}{|c|}{ Chaetodontidae } \\
\hline & Chaetodon striatus Linnaeus, 1758 & 0,033 & SI \\
\hline \multicolumn{4}{|c|}{ Pomacanthidae } \\
\hline & Holacanthus ciliaris (Linnaeus, 1758) & 0,008 & SI \\
\hline & Holacanthus tricolor (Bloch, 1795) & 0,384 & SI \\
\hline & Pomacanthus paru (Bloch, 1787) & 0,008 & SI \\
\hline \multicolumn{4}{|c|}{ Cirrhitidae } \\
\hline & Amblycirrhitus pinos (Mowbray, 1937) & 0,070 & MI \\
\hline \multicolumn{4}{|c|}{ Pomacentridae } \\
\hline & Abudefduf saxatilis (Linnaeus, 1758) & 42,806 & $\mathrm{O}$ \\
\hline & Stegastes pictus (Castelnau, 1855) & 0,004 & $\mathrm{TH}$ \\
\hline & Chromis multilineata (Castelnau, 1855) & 75,405 & PL \\
\hline
\end{tabular}




\begin{tabular}{|c|c|c|c|}
\hline Família & Espécie & $\operatorname{Fr}(\%)$ & C.T \\
\hline \multicolumn{4}{|c|}{ Labridae } \\
\hline & Bodianus rufus (Linnaeus, 1758) & 29,651 & MI \\
\hline & Clepticus brasiliensis (Heiser, Moura \& Robertson, 2000) & 24,145 & PL \\
\hline & Halichoeres brasiliensis (Bloch, 1791) & 0,087 & MI \\
\hline & Halichoeres dimidiatus (Agassiz, 1831) & 0,153 & MI \\
\hline & Halichoeres poeyi (Steindachner, 1867) & 0,041 & MI \\
\hline & Thalassoma noronhanum (Boulenger, 1890) & 0,285 & PL \\
\hline \multicolumn{4}{|c|}{ Scaridae } \\
\hline & Scarus trispinosus Valenciennes, 1840 & 0,008 & $\mathrm{RH}$ \\
\hline & Sparisoma axillare (Steindachner, 1878) & 0,231 & $\mathrm{RH}$ \\
\hline & Sparisoma amplum (Ranzani, 1842) & 0,004 & $\mathrm{RH}$ \\
\hline & Sparisoma radinas (Valenciennes, 1840) & 0,008 & $\mathrm{RH}$ \\
\hline & Sparisoma frondosum (Agassiz, 1831) & 0,016 & $\mathrm{RH}$ \\
\hline \multicolumn{4}{|c|}{ Gobiidae } \\
\hline & Coryphopterus glaucofraenum Gill, 1863 & 0,012 & $\mathrm{O}$ \\
\hline & Elacatinus figaro Sazima, Moura \& Rosa, 1997 & 0,070 & MI \\
\hline \multicolumn{4}{|c|}{ Microdesmidae } \\
\hline & Ptereleotris randalli Gasparini, Rocha \& Floeter, 2001 & 0,037 & PL \\
\hline \multicolumn{4}{|c|}{ Ephippidae } \\
\hline & Chaetodipterus faber (Broussonet, 1782) & 1,691 & SI \\
\hline \multicolumn{4}{|c|}{ Acanthuridae } \\
\hline & Acanthurus bahianus Castelnau, 1855 & 0,054 & $\mathrm{O}$ \\
\hline & Acanthurus chirurgus (Bloch, 1787) & 0,548 & $\mathrm{O}$ \\
\hline & Acanthurus coeruleus (Bloch \& Scheneider, 1801) & 0,726 & $\mathrm{RH}$ \\
\hline \multicolumn{4}{|c|}{ Sphyraenidae } \\
\hline & Sphyraena barracuda (Edwards, 1771) & 0,004 & $\mathrm{P}$ \\
\hline \multicolumn{4}{|c|}{ Scombridae } \\
\hline & Scomberomorus regalis (Bloch, 1793) & 0,181 & $\mathrm{P}$ \\
\hline \multicolumn{4}{|c|}{ Balistidae } \\
\hline & Balistes vetula Linnaeus, 1758 & 0,004 & MI \\
\hline \multicolumn{4}{|c|}{ Monacanthidae } \\
\hline & Cantherhines pullus (Linnaeus, 1758) & 0,012 & $\mathrm{O}$ \\
\hline \multicolumn{4}{|c|}{ Tetraodontidae } \\
\hline & Canthigaster figueiredoi Moura \& Castro, 2002 & 0,045 & SI \\
\hline
\end{tabular}

\section{Relações ecológicas}

Atividades de limpeza

Algumas áreas do naufrágio funcionavam como estação de limpeza, onde foram observados indivíduos de Elacatinus figaro realizando atividade de limpeza em indivíduos de Sparisoma axillare e Cephalopholis fulva (Figura 03-B e C) em um mesmo momento, onde as espécies clientes revezavam-se assumindo uma postura estática enquanto os limpadores realizavam a limpeza. 
Em outra observação envolvendo limpadorcliente, foram vistos indivíduos jovens de $B$. rufus realizando limpeza em um indivíduo de $\mathrm{H}$. aurolineatum (Figura 03-D), que com a aproximação do limpador assumia uma postura vertical com a cabeça para baixo e nadadeiras abertas, permanecendo imóvel durante a limpeza. Jovens de B. rufus, medindo entre $3-5$ $\mathrm{cm}$, também foram observados realizando limpeza em indivíduos de $C$. multilineata, que também assum- iram uma postura na posição vertical com a cabeça voltada para baixo, e em um indivíduo de Anisotrmus surinamensis adulto (entre $35-40 \mathrm{~cm}$ ) próximo ao casario da embarcação. Foi observada também simbiose de limpeza envolvendo $A$. saxatilis e $B$. rufus em Eretmochelys imbricata (Tartaruga-de-pente), onde os limpadores investiam sobre o casco coberto por algas e pequenos invertebrados sésseis (Figura 03-A).

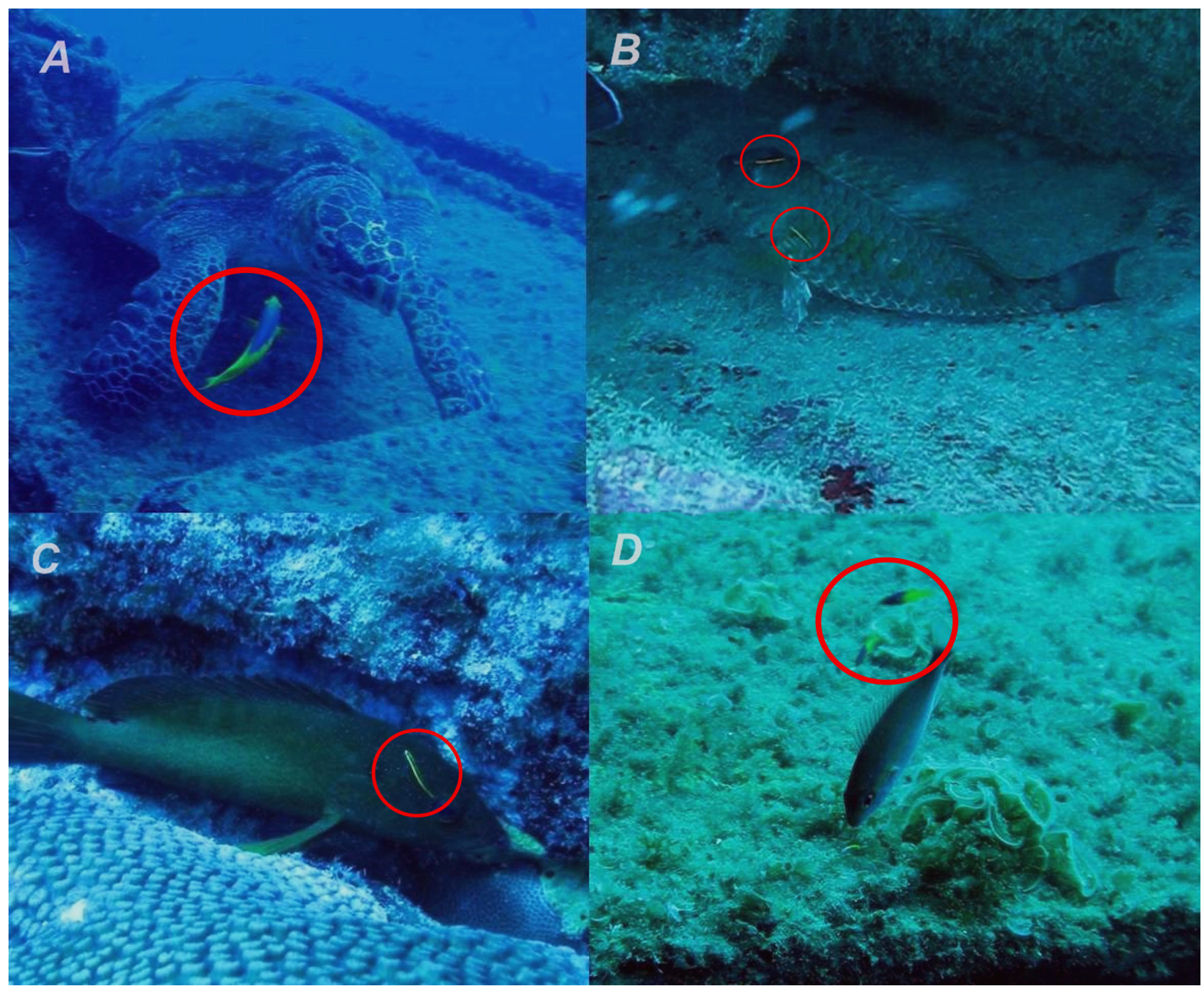

Figura 03: Simbiose de limpeza entre B. rufus e E. imbricata (A); E. figaro e exemplares de S. axillare e C. fulva (B e C); entre B. rufus e H. aurolineatum (D), no naufrágio Gonçalo Coelho, Serrambi/PE. (Crédito fotografia A: Danielle Viana).

\section{Comportamento seguidor e formação de cardume misto}

Durante as observações foram registrados indivíduos das espécies Halichoeres dimidiatus seguindo indivíduos da espécie Mulloidichthys martinicus (Figura 04-D) enquanto a mesma revirava o substrato em busca de alimento. Essas duas espécies frequentemente avistadas em comportamento seguidor, mostram-se bastante oportunistas, provavelmente pelo seu hábito alimentar. Foi observado também um indivíduo de $\mathrm{B}$. rufus seguindo um indivíduo da espécie Dasyatis americana (Figura 04-C) que buscava abrigo, e seguindo também um Acanthurus chirurgus (Figura 04-B) enquanto forrageava o fundo em busca de alimento. Foram observados indivíduos de Lutjanus synagris seguindo indivíduos de Haemulon parra próximo ao convés da embarcação (Figura 04-A). 


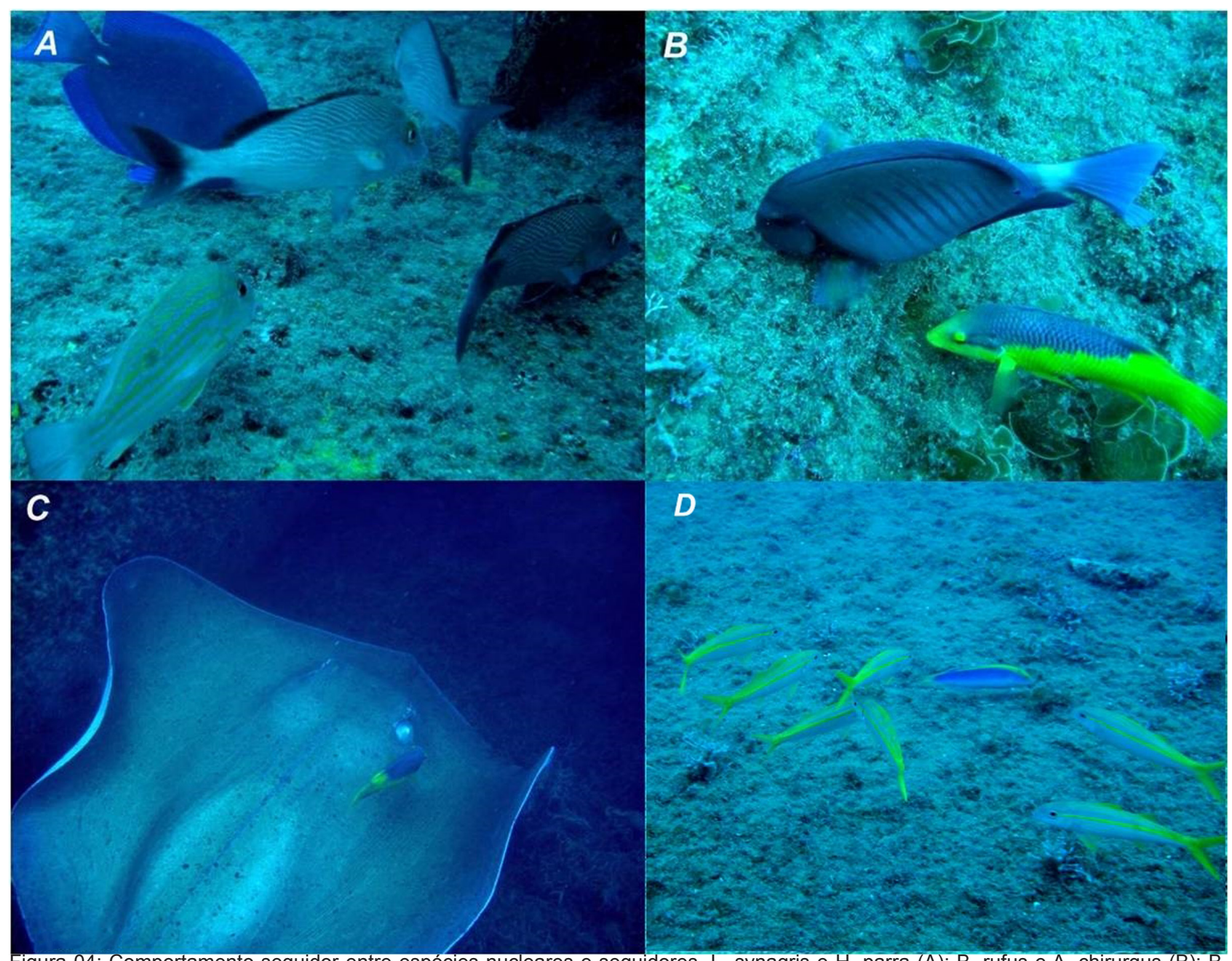

Figura 04: Comportamento seguidor entre espécies nucleares e seguidores. L. synagris e H. parra (A); B. rufus e A. chirurgus (B); B. rufus e D. americana (C); M. martinicus e espécie seguidora, P. maculatus e O. crysurus (D) no naufrágio Gonçalo Coelho, Serrambi/PE. (Crédito fotografia C: Dráusio Véras).

A formação de cardume misto foi bastante observada ao longo dos mergulhos. Nas observações, indivíduos da espécie $\mathrm{H}$. parra e Lutjanus alexandrei (Figura 05-B), eram vistas com bastante frequência em formação mista. Provavelmente por ambas estarem em cardumes numericamente pequenos, tenham recorrido à formação de um cardume misto para parecerem mais numerosas em relação aos predadores. Indivíduos de C. multilineata e C. brasiliensis com comprimento médio de $5 \mathrm{~cm}$ também foram observados formando grandes cardumes mistos (Figura 05-A). Nessa fase de vida, essas duas espécies apresentam características morfológicas muito semelhantes. Provavelmente essa postura adotada seja parte da estratégia de defesa devido ao pequeno tamanho dos indivíduos. Além disso, em outra observação, em um mesmo momento três espécies de duas famílias distintas foram avistadas em um pequeno cardume misto, sendo $M$. martinicus a mais numerosa, um outro indivíduo de $\mathrm{O}$. crysurus e um de Pseudupeneus maculatus. Provavelmente por estarem também em números reduzidos, se agruparam formando um cardume maior (Figura 05-C).

\section{Predação}

Eventos de predação também foram observados. Foram registrados eventos envolvendo Caranx crysos, Caranx latus e Scomberomorus regalis predando cardumes de D. macarellus. Outro evento relacionado à predação, nesse caso o registro de oofagia (indivíduos se alimentando de ovos de outras espécies), envolveu um indivíduo de M. martinicus alimentando-se de ovos de $A$. saxatilis depositados ao lado da estrutura do naufrágio (Figura 06). 


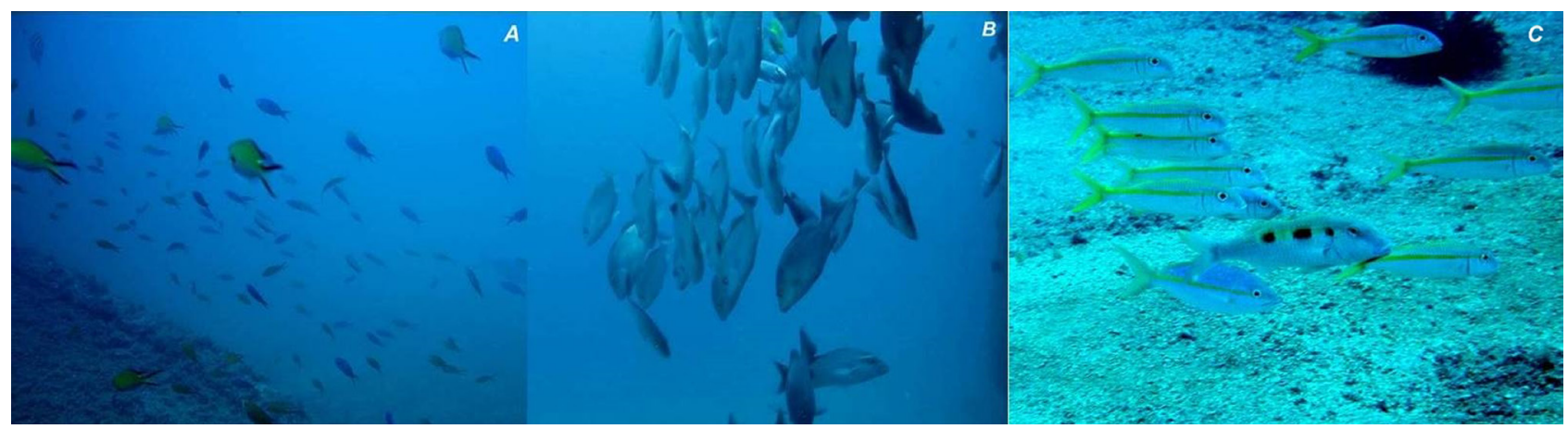

Figura 05: Formação de cardume misto. C. brasiliensis e C. multilineata (A); H. parra e L. alexandrei (B); P. maculatus, M. martinicus e O. crysurus $(\mathrm{C})$ no naufrágio Gonçalo Coelho, Serrambi/PE. (Crédito fotografia C: Dráusio Véras).

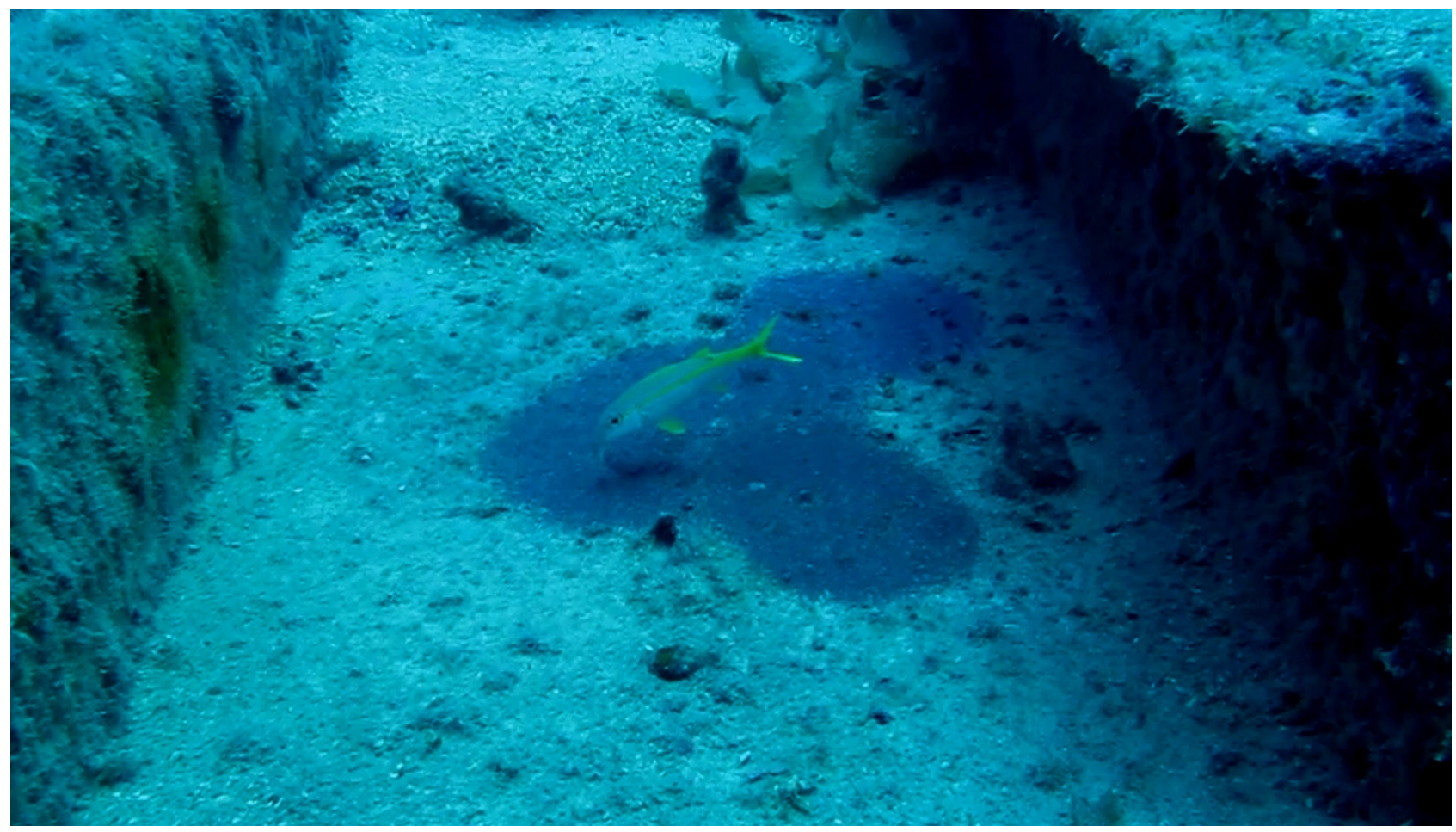

Figura 06: Ocorrência de oofagia. M. martinicus predando ovos de A. saxatilis no naufrágio Gonçalo Coelho, Serrambi/PE

\section{DISCUSSÃO}

\section{Estrutura trófica}

A organização trófica da ictiofauna do Gonçalo Coelho mostrou que os peixes planctívoros foram os mais abundantes em relação à distribuição total, padrão já observado em estudos realizados em ambientes de recifes artificiais por Rilov \& Benayahu (2000).

Segundo Floeter et al. (2007), um dos fatores que podem determinar a ocorrência e abundância de espécies de peixes recifais planctívoras é o fator distância da costa, reforçando a ideia de que quanto mais próximo do limite da plataforma continental o recife estiver inserido, maior a disponibilidade de plâncton no mesmo. Algo que já foi observado em estudos realizados por Thressher \& Colin (1986) e Feitoza (2005). No caso dos recifes artificiais, os mesmos podem funcionar como barreiras para correntes marinhas, criando um afloramento de nutrientes e alimentos que estão depositados no leito marinho. Por essa razão espécies plactívoras geralmente são abundantes nessas áreas (Linquist \& Pietrafesa, 1989), sendo observados esses mesmos padrões no naufrágio Gonçalo Coelho, com a predominância das espécies $C$. multilineata, $D$. macarellus e C. brasiliensis.

A segunda classe trófica mais abundante foi a dos predadores de invertebrados móveis. Essa abundância pode estar diretamente relacionada às características físicas do naufrágio que apresenta um comprimento de 64 metros, porém baixa rugosidade e baixo número de tocas disponíveis. Segundo Dominici-Arosemena \& Wolf (2005), a abundância e 
a diversidade de predadores de invertebrados móveis tende a aumentar de áreas mais protegidas para áreas mais expostas.

A terceira classe mais abundante, a dos peixes onívoros, esteve representada principalmente pela família Pomacentridae, com A. saxatilis sendo a espécie mais representativa. Essa abundância pode estar relacionada ao hábito alimentar oportunista da espécie e a disponibilidade de alimento encontrada no naufrágio. Dentre as famílias de peixes recifais, a família Pomacentridae é umas das mais relevantes, apresentando altas densidades, diversidade e abundância (Pough et al., 2003).

\section{Relações ecológicas}

Relações ecológicas entre as comunidades de peixes recifais são de fundamental importância no equilíbrio e saúde da comunidade ictíicas do recife artificial (Wilson \& Wilson, 1992, DeLoach, 1999).

A simbiose de limpeza é umas das associações, envolvendo alimentação, mais importante em comunidades ictíicas recifais, podendo envolver uma grande diversidade de espécies (Hobson, 1971, Losey, 1971). As espécies que realizam essa atividade são essenciais na remoção de ectoparasitas, tecido doente e muco proveniente do corpo de outros peixes (DeLoach, 1999). São descritas aproximadamente 100 espécies com hábito limpador, distribuídas em 19 famílias, podendo pertencer a categoria dos limpadores obrigatórios (durante todo seu ciclo de vida) e limpadores facultativos (quando em sua fase juvenil) (Côté, 2000). Durante os mergulhos realizados, destacou-se o gênero Elacatinus (Gobiidae), como um dos mais observados realizando simbiose de limpeza (Tabela 03). Nas observações, indivíduos de E. figaro foram observados realizando limpeza em clientes das espécies $S$. axillare e C. fulva. Essa relação do E. figaro com $C$. fulva foi frequentemente observada por Randall (1967) em Fernando de Noronha/PE. Já a relação com a família Scaridae foi observada por Sazima et al. (2000) nas Ilhas Anchieta e Vitória - SP, na Laje de Santos - SP, e por Campos \& Sá-Oliveira (2011) nos recifes de coral dos Parrachos de Muriú/RN.

Tabela 03 - Eventos observados envolvendo simbiose de limpeza, com respectivos limpadores e clientes, na ictiofauna associada ao Naufrágio Gonçalo Coelho, Serrambi/PE. Categoria funcional segundo Côté, (2000) e categoria trófica segundo Ferreira et al. (2004).

\begin{tabular}{lccl}
\hline Limpador & Categoria funcional & Categoria trófica & Cliente \\
\hline Elacatinus fígaro & Limpador obrigatório & MI & $\begin{array}{l}\text { Sparisoma axillare } \\
\text { Cephalopholis fulva }\end{array}$ \\
\hline & & & $\begin{array}{l}\text { Anisotremus surinamensis } \\
\text { Bodianus rufus }\end{array}$ \\
& Limpador facultativo & MI & $\begin{array}{l}\text { Haemulon aurolineatum } \\
\text { Chromis multilineata }\end{array}$ \\
& & & Eretmochelys imbricata \\
\hline Abudefduf saxatilis & Limpador facultativo & $\mathrm{O}$ & Eretmochelys imbricata \\
\hline
\end{tabular}

B. rufus foi outra espécie observada em simbiose de limpeza com indivíduos de A. surinamensis, H. aurolinetam, C. multilineta e E. imbricata. Essas relações aconteceram principalmente envolvendo jovens de B. rufus. Segundo Lieske \& Myers (1999) e Côté (2000), algumas espécies exercem essa função apenas quando jovens, como foi observado. A associação com $A$. surinamensis ocorreu na coluna d'água, acima do casario da embarcação, provavelmente devido ao tamanho corpóreo do A. surinamensis. Nas observações envolvendo $B$. rufus realizando limpeza em indivíduos de $\mathrm{C}$. multilineata e $\mathrm{H}$. aurolineatum, as espécies cliente apresentaram o mesmo padrão de comportamento, assumindo uma posição vertical com a cabeça voltada para baixo e abrindo as nadadeiras como sinal de aviso aos limpadores, corroborando com Sazima et al. (2006) que observou o mesmo padrão de comportamento.

Em outra observação, exemplares de B. rufus e A. saxatilis foram vistos se alimentando de pequenos organismos e algas no casco de uma E. imbricata. Sazima et al. (2009) observou essas relações envolvendo as espécies citadas em Fernando de Noronha/ PE e no naufrágio Pirapama/PE. Comportamento de pastagem e limpeza em tartarugas marinhas foram registrados em diversos estudos para o Atlântico e Pacífico (Booth \& Peters, 1972, Losey et al., 1994, Sazima et al., 2004).

O comportamento do tipo seguidor foi observado entre algumas espécies (Tabela 04), o mesmo consiste em uma espécie nuclear revirar o substrato em busca de sua presa e as espécies denominadas seguidoras aproveitam os restos de sua captura (Leitão et al. 2007, DeLoach, 1999). Essas interações envolveram espé- 
cies da família Mulidae (M. martinicus) e Labridae (B. rufus) tanto como seguidoras e nucleares, L. synagris apenas como seguidor $\mathrm{e} D$. americana, $A$. chirurgus $e$ H. parra apenas nucleares. Segundo Carvalho-Filho (1999), as espécies nucleares habitam geralmente fundos rochosos, bolsões de areia ou cascalho e bancos de algas. Essas características são apontadas como o principal motivo para a atração de espécies oportunistas (Dias, 2007).

A formação de cardume misto pode estar associada à alimentação, bem como aos comportamentos seguidor e de defesa (Tabela 04). As espécies normalmente apresentam este tipo de comportamento, seja para ter acesso aos recursos defendidos por territorialistas, para diminuir o tempo de busca por recursos ou proporcionar maior proteção contra a predação (Ehrlich \& Eherlich, 1973, Itzkowitz, 1977, Debrot \& Myberg, 1988, Ward et al., 2002, Carvalho et al., 2007). No naufrágio Gonçalo Coelho, essas características ficaram evidentes, principalmente entre indivíduos de C. brasiliensis e C. multilineata, entre H. parra e L. alenxandrei e formação de cardume misto entre P. maculatus, M. martinicus e $\mathrm{O}$. crysurus. Padrões semelhantes foram observados por Krajewski et al. 2004, que observou formação de cardume misto entre $\mathrm{M}$. martinicus e $\mathrm{H}$. chrysargyreum com função de mimetismo de proteção e por Braga et al. 2009 que observou três espécies da família Haemulidae formando cardume misto relacionados a abundância de cada espécie e a presença de predadores.

Os eventos de predação (Tabela 04) envolveram principalmente espécies de hábito alimentar piscívoro, como espécies das famílias Carangidae e Scombridae, que normalmente são atraídas pela abundância de espécies planctívoras que visitam o naufrágio em busca de alimento, conforme relatado por Linquist \& Pietrafesa (1989).

Tabela 04. Eventos observados envolvendo comportamento seguidor, formação de cardume misto e predação na ictiofauna associada ao Naufrágio Gonçalo Coelho, Serrambi/PE.

\begin{tabular}{lll}
\hline \multicolumn{1}{c}{ Espécie nuclear } & Seguidor & Comportamento \\
\hline Haemulon parra & Lutjanus synagris & Acompanhando o indivíduo \\
Bcanthurus chirurgus & Bodianus rufus & $\begin{array}{l}\text { Alimentando-se no substrato } \\
\text { revirado } \\
\text { Acompanhando o indivíduo }\end{array}$ \\
Mulloidichthys martinicus & Halichoeres dimidiatus & $\begin{array}{l}\text { Alimentando-se no substrato } \\
\text { revirado }\end{array}$ \\
Clepticus brasiliensis & Chromis multilineata & Formação de cardume misto \\
Haemulon parra & Lutjanus alexandrei & Formação de cardume misto \\
Mulloidichthys martinicus & Pseudupeneus maculatus & Formação de cardume misto \\
\hline Predador & Presurus chrysurus & Comportamento \\
\hline Caranx crysos & Decapterus macarellus & Predação \\
Caranx latus & Decapterus macarellus & Predação \\
Scomberomorus regalis & Decapterus macarellus & Predação \\
\hline Mulloidichthys martinicus & Abudefduf saxatilis & Oofagia \\
\hline
\end{tabular}

Araújo et al. (2004) relata a ocorrência de oofagia envolvendo E. figaro predando ovos de A. saxatilis em ambiente natural de poças de maré no litoral de Pernambuco. Evento semelhante foi observado no presente estudo, porém envolvendo também a predação de ovos de A. saxatilis, mas por indivíduos de M. martinicus.

A ocorrência dessas relações ecológicas, reforçam o quanto estruturas artificiais exercem funções similares as encontradas em ambientes recifais naturais e desempenham funções importantes para a manutenção e controle das comunidades ictíicas associadas as mesmas.

Em relação ao naufrágio Gonçalo Coelho, a observação de eventos envolvendo predação, simbiose de limpeza, assentamento de larvas, registro de indivíduos juvenis e ocorrência de espécies listadas como vulneráveis e ameaçadas, por exemplo Scarus trispinosus e Epinephelus itajara, mostra que o mesmo vem desempenhando papel ecológico importante junto às comunidades de peixes próximas. 
Espera-se que essas informações possam incentivar novos estudos com estruturas artificiais para a costa nordeste do Brasil e contribuir de forma positiva para a elaboração de medidas que venham regulamentar e assegurar o uso adequado de estruturas artificias como importante ferramenta na criação, implantação e gestão de áreas de proteção à vida marinha como também auxiliar na recuperação de ambientes degradados pela ação antrópica.

\section{REFERÊNCIAS}

Araújo, M. E.; Paiva, A.C.G. \& Mattos, R.M.G. 2004. Predação de ovos de Abudefduf saxatilis (Pomacentridae) por Elacatinus figaro (Gobiidae) em poças de maré, Serrambi, Pernambuco. Tropical Oceanography. 32(2): 135-142.

Arnal, C.; Côté, I.M. \& Morand, S. 2001. Why clean and be cleaned? The importance of client ectoparasites and mucus in a marine cleaning symbiosis. Behavioral Ecology and Sociobiology. 57: 1-7.

Braga, M. R.; Braga, R. R. \& Aranha, J. M. R. 2009. Registro de cardume misto de espécies da família Haemulidae nas Ilhas Itacolomis, Santa Catarina, Brasil. Estudos Biológicos. 31(73/74/75):141-143.

Booth, J. \& Peters, J.A. 1972. Behavioural studies on the green turtle (Chelonia mydas) in the sea. Animal Behaviour. 20: 808-812.

Campos, C.E.C. \& Sá-Oliveira, J.C. 2011. Atividade de limpeza e clientes de Elacatinus figaro (Pisces: Gobiidae) nos recifes de coral dos Parrachos de Muriú, Nordeste do Brasil. Biota Neotropical. 11(1): 47-51.

Carvalho, D. C.; Corneta, C. M. \& Uieda, V. S. 2005. Schooaling behavior of Mugil curema (Perciformes: Mugilidae) in an esturay in southeastern Brazil. Neotropical Ichthyology. 5(1):81-83.

Carvalho-Filho, A. 1999. Peixes: costa brasileira. $3^{\circ}$ edição. Editora Meiro, São Paulo, 320p.

Cavalcanti, L.B. \& Kempf, M. 1970. Estudo da plataforma continental na área de Recife (Brasil). II. Metodologia e Hidrologia. Trabalhos Oceanográficos da Universidade Federal de Pernambuco. 9(11):149-158.

Collin, M.R. \& Sedberry, G.R. 1991. Status of vermilion snapper and red porgy stocks off South-Carolina. Transactions of the American Fisheries Society. 120(1): 116-120.

Côté, I.M. 2000. Evolution and ecology of cleaning symbioses in the sea. Oceanography and Marine Biology - An Annual Review. 38:311-355.

DeLoach, N. 1999. Reef fish behavior: Florida, Caribbean, Bahamas. $1^{\circ}$ Edição. New World Publications, inc. 359p.
Debrot, A.O. \& Myrberg, Jr.A. A. 1988. Intraspecific avoidance as a proximate cause for mixedspecies shoaling by juveniles of western Atlantic surgeonfish, Acanthurus bahianus. Bulletin of Marine Science. 43: 104-106.

Dias, T.L.P. 2007. What do we know about Anisotremus moricandi (Teleostei: Haemulidae), an endangered reef fish? Biota Neotropical. May/Aug. 7(2).

Dominici-Arosemena, A. \& Wolff, M. 2005. Reef fish community structure in Bocas del Toro (Caribbean, Panamá): Gradients in habitat complexity and exposure. Caribbean Journal of Science. 41(3): 613-637.

Ehrlich, P.R. \& Eherlich, A.H. 1973. Coevolution: Heterotypic schooling in Caribbean reef fishes. American Naturalist. 107:157-160.

Ferreira, C.E.L.; Floeter, S R.; Gasparini, J.L.; Ferreira, B.P. \& Joyeux, J.C. 2004. Trophic structure patterns of Brazilian reef fishes: a latitudinal comparison. Journal of Biogeography. 31:1093-1106.

Floeter, S.R.; Krohling, W.; Gasparini, J.L.; Ferreira, C.E.L. \& Zalmon, I.R. 2007. Reef fish community structure on coastal islands of the southeastern Brazil: the influence of exposure and benthic cover. Environmental Biology of Fishes. 78:147-160.

Hobson, E.S. 1971. Cleaning symbiosis among California inshore fishes. Fishery Bulletin. 69:491-523.

Itzkowitz, M. 1977. Social dynamics of mixed-species groups of Jamaican reef fishes. Behavioral Ecology and Sociobiology, 2:361-384.

Jr. Palazzo, J. T. 2011. Naufrágios e Pontos de Mergulho de Fernando de Noronha, Recife e Maceió. Cultura Sub, São Paulo, p.162-164.

Keenleyside, M. H. A. 1979. Diversity and adaptation in fish behavior. Springer-Verlag. New York, 208p.

Krajewski, J. P.; Bonaldo, R. M.; Sazima, C. \& Sazima, I. 2004. The association of the goatfish Mulloidichthys martinicus with the grunt Haemulon chrysargyreum: an example of protective mimicry. Biota Neotropica. 4(1):1-4.

Leitão, R.P.; Caramaschi, E.P. \& Zuanon, J. 2007. Following food clouds feeding association between a minute loricariid and characidiin species in an Atlantic Forest stream Southeastern Brazil. Neotropical Ichytyology. 5(3): 307-310.

Lindquist, D. G. \& Pietrafesa, L. J. 1989. Current vortices and fish aggregations: the current field and associated fishes around a tugboat in Onslow Bay, North Carolina. Bulletin of Marine Science. 44:533-544.

Liesk, E. \& Myers, R. 1999. Caribbean, Indian Ocean and Pacific Ocean including the Red Sea. Princeton University. Press. New Jersy. 400p.

Losey, G.S.J. 1971. Communication between fishes 
in cleaning symbiosis. In Aspects of biology of symbiosis, T.C. Cheng (Ed.). University Park Press, Baltimore. 45-76.

Losey, G.S.; Balazs, G. H. \& Privitera, L. A. 1994. Cleaning symbiosis between the wrasse, Thalassoma duperrey, and the green turtle, Chelonia mydas. Copeia (3): 684-690.

Pough, F.H.; Janis, C.M. \& Heiser, J. B. 2003. A vida dos vertebrados. $3^{\circ}$ edição. Editora Atheneu. São Paulo, 596p.

Randall, J.E. 1967. Three new species and six new records of small serranoid fishes from Curaçao and Puerto Rico. Studies on the Fauna of Curaçao and other Caribbean Islands. 80(19):77-110.

Rilov, G. \& Benayahu, Y. 2000. Fish assemblage on natural versus vertical artificial reefs: the rehabilitation perspective. Marine Biology. 136: 931-942.

Sazima, I.; Sazima, C.; Francini-Filho, R. B. \& Moura, R. L. 2000. Daily cleaning activity and diversity of clients of the barber goby, Elacatinus figaro, on rocky reefs in Southeastern Brazil. Environmental Biology of Fishes. 59(1), 69-77.

Sazima, I.; Grossman, A. \& Sazima, C. 2004. Hawksbill turtles visit moustached barbers: cleaning symbiosis between Eretmochelys imbricata and the shrimp Stenopus hispidus. Biota Neotropica, 4(1): 1-6.

Sazima, C.; Krajewski, J. P.; Bonaldo, R. M. \& Guimarães-Jr, P. R. 2006. The goatfish Pseudupeneus maculatus and its follwer fishes at an oceanic islands in the tropical West Atlantic. Journal of Fish Biology. 69:883-891.

Sazima, C.; Grossman, A. \& Sazima, I. 2009. Turtle cleaners: reef fishes foraging on epibitions of sea turtles in the tropical Southwestern Atlantic, with a summary of this association type. Neotropical Ichthyology. 8(1).

Spalding, M.D.; Ravilious, C. \& Green, E.P. 2001. World Atlas of Coral Reefs. University of California . Press. 424 pp.
Seaman, W.J. \& Jensen, A.C. 2005. Purposes and practices of artificial reef evaluation. In: Seaman, W. J. (ed). Artificial Reef Evaluation: With Application to Natural Marine Habitats. CRC Press. Florida. 1-20 pp.

Seaman, W.Jr. 2000. Artificial reef evaluation: with application to natural marine habitats. CRC Press. Florida. 246p.

Sale, P.F. 1980. The ecology of fishes on coral reefs. Oceanography Marine Biology. Annual review 18: 367-421.

Sale, P. F.; Cowem, R. K.; Danilowicz, B.S.; Jones, P.G.; Krtzer, J.P.; Lindeman, K.C.; Planes, S.; Polunin, N.V.C.; Russ, G.R.; Sadovy, Y.J. \& Steneck, R.S. 2005. Critical science gaps impede use of no-take fishery reserves. Trends Ecology Evolution. 20: 74-80.

Thresher, R.E.\& Colin, P.L. 1986. Trophic structure, diversity and abundance of fishes of the deep reef (30-300 $\mathrm{m})$ at Enewetak, Marshall Islands. Bulletin of Marine Science 38:253-272.

Ward, A.J.W.; Axford, S. \& Krause, J. 2002. Mixedspecies shoaling in fish: the sensory mechanisms and costs of shoal choice. Behavioral Ecology and Sociobiology, 52: 182-187.

Wilson, R. \& Wilson, J. Q. 1992. Pisces guide to watching fishes: understanding coral reef fish behavior. Lonely Planet Publications. Hawthorn, $275 p$.

Submetido: Novembro/2013

Revisado: Junho/2015

Aceito: Agosto/2015 